
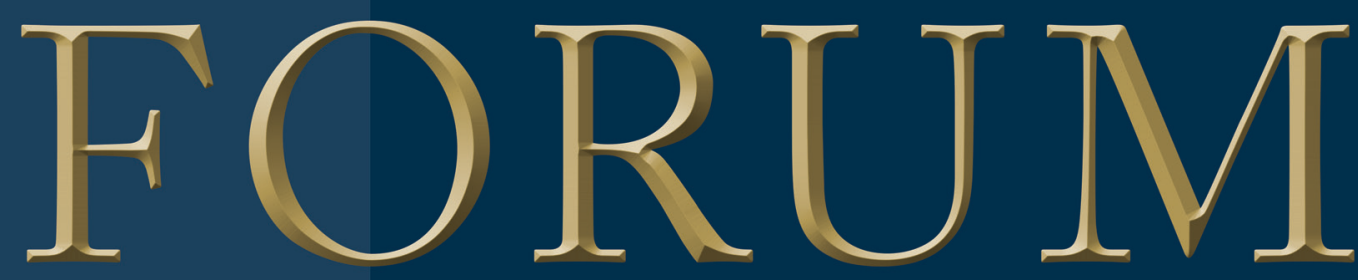

VOLUME 27 | NUMBER 5

SEPTEMBER/OCTOBER

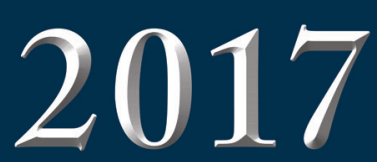

HAIR TRANSPLANT FORUM INTERNATIONAL

IN THIS ISSUE

Prophylactic

Antibiotics in Hair

Transplantation Surgery:

Recommendations to

Avoid Use Except in

Limited Circumstances

Life-Threatening Infection After Hair

Transplantation Surgery

Innovation and Quality in Hair Restoration

Surgery

Combining Medical and Scientific

Innovation in Hair

"Cloning"
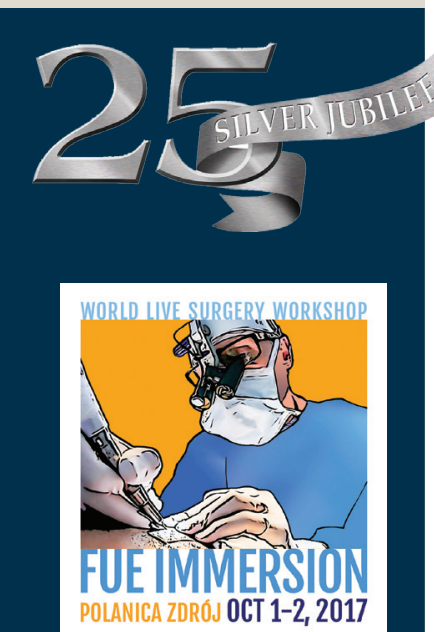

Register Today! www.25thannual.org

\section{Hair Transplant in the Age of MRSA}

\section{Sara Wasserbauer, MD, FISHRS I Walnut Creek, California, USA I}

drwasserbauer@californiahairsurgeon.com

The biggest threat to your practice is not a disruptive new surgical technology or medical treatment, it's MRSA. We live and practice medicine in the post-antibiotic era. Our field of elective hair transplant surgery is only possible due to the discovery of penicillin in 1928 by Alexander Fleming and the subsequent expansion of our biological warfare armamentarium. Without effective strategies to control the biological threats we live with, surgical intervention of any type is an inadvisable risk.

Now, like it or not, that existence is threatened by the very weapons we created. Worldwide, rates of antibiotic-resistant bacterial infections have steadily climbed, particularly in the past decade. In 2013, the U.S. Centers for Disease Control (CDC) categorized the threats based on level of concern: "urgent," "serious," and "concerning." The urgent threats are not commonly encountered in the normal course of a

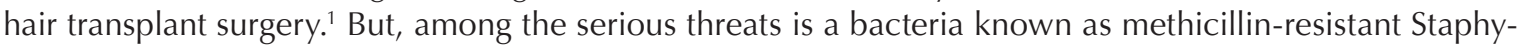
lococcus aureus (MRSA), and it is a real and emerging threat in our specialty. ${ }^{2}$

\section{HOW DID WE GET HERE? MRSA HISTORY LESSON}

Staphylococcus (S.) aureus is ubiquitous on our skin and mucous membranes, and usually it does not cause any harm. It is estimated that $25 \%-35 \%$ of healthy humans carry S. aureus in this post-antibiotic era. It would be a mistake, however, to assume that S. aureus is benign. If you look at data from the pre-antibiotic era and into the 1940s, S. aureus bacteremia was usually fatal.

MRSA is the term that describes strains of $\mathrm{S}$. aureus that have acquired antibiotic resistance either through overuse of antibiotics for inappropriate treatment indications (like treating a viral infection with antibiotics), or through incomplete treatment leading to the selection of antibiotic-resistant strains. Excessive perioperative antibiotic use by physicians also likely played a role in initiating these strains.

MRSA was first identified in the 1960s and at that time it was an iatrogenic infection; that is, it was typically found in hospitals, nursing homes, or isolated from medical personnel. Unless it gained access inside the body, a person's skin was an excellent barrier and his or her immune system was very good at fighting it off. Plus, if MRSA infected a patient, switching to an antibiotic that the strain still WAS susceptible to usually still cleared the infection.

In the late 1990s, the situation changed. This little bug moved from a healthcare-associated illness to one that can be acquired from one's community. Patients and medical personnel without active infection symptoms would carry the resistant strain outside of the hospitals and nursing homes. Individuals were commonly colonized in the nose and on the skin so a simple sneeze or touch of the hand could transmit the bacteria. The more the bacterial strains spread, the more the strains got the chance to develop resistance to new antibiotics, until one by one whole antibiotic classes started to fail. In 1999, we heard the first reports of children dying due to resistant MRSA infections.

Over the past 30 years, no new major antibiotics have been developed, so now we are down to our last effective treatments. ${ }^{4}$ In 2002, the first reported case of vancomycin-resistant S. aureus in the United States (Figure 1) was
FIGURE 1. Magnified 20,000x, this colorized scanning electron micrograph (SEM) depicts a grouping of methicillin-resistant Staphylococcus aureus (MRSA) bacteria. Photo credit: Public Health Image Library: https://www.cdc.gov/mrsa/community/

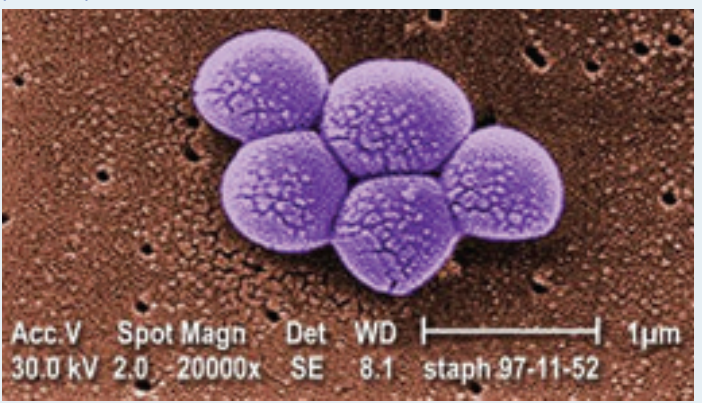
photos/photo-mrsa-13.html 
established. Since vancomycin was one of the last antibiotics we had to treat infections of this kind, the isolation of this strain of bacteria served as a wake-up call that we are headed toward a post-antibiotic era in which elective surgeries like hair transplantation may give you an untreatable infection. ${ }^{5}$

\section{A problem for all hair surgeons worldwide}

Worldwide rates of MRSA can be tracked to antibiotic overuse and misuse. As such, excellent and robust health care systems with an abundance of resources are just as vulnerable to MRSA spread as those in less developed countries where less clean facilities and fewer trained staff exist. In fact, MRSA rates are just as high in industrialized "First World" countries as they are in less economically fortunate ones, although the various strains may be different. ${ }^{6}$ (See Figure 2.)

FIGURE 2. MRSA infection rates by country. Graph obtained from http://www.cddep. org/tool/mrsa_infection_rates_country

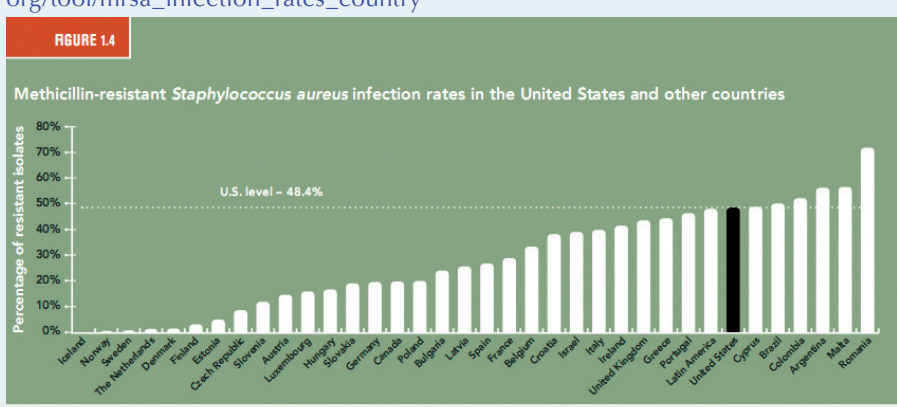

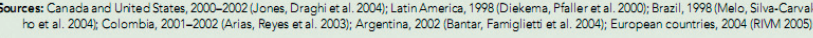

No matter which strain you are dealing with, MRSA can cause a range of infections, including in skin and surgical wounds. This is why out of the list of infectious agents considered "serious" by the U.S. Centers for Disease Control, MRSA is the one that will most affect hair surgeons. MRSA has the potential to cause problems with hair transplant patients even more so than with other forms of elective surgery because the areas with hair can never be completely sterilized. Additionally, there is the problem of asymptomatic carriers. Staff and patients alike may be colonized with MRSA without knowing it, and may easily spread it to each other due to the close proximity during a hair transplant surgery.

Medical tourism to obtain low-cost hair transplantation (or other surgeries) is an additional risk for new MRSA infections and fosters the spread of individual strains along the most heavily traveled air routes. ${ }^{7}$ Epidemiological experts can track certain strains or resistant bacteria to specific regions worldwide, and often the new host (the medical tourism patient) is less able to fight off the novel infection than is the native population where the surgery was performed. The native staff also may be unaware of the problems that they cause, since they never see their patients again in most cases. Additionally, appropriate treatment may not be as promptly administered and follow-up care is interrupted, worsening the plight of the patient.

Studies show that about 1 in 3 people carry staph in their nose, usually without any illness. Two in 100 people carry MRSA. No data shows the total number of people who get MRSA skin infections in the community, and data that breaks out MRSA rates with scalp surgeries likewise does not exist at this time. ${ }^{8}$ Given the proximity of the nose (the

most commonly colonized area in asymptomatic carriers) to the scalp, hair surgeries in general are at higher risk. Compounding the danger is that hair surgery is often performed as an outpatient procedure, with minimally trained or licensed personnel who may not adhere to sterile techniques as rigorously as more highly trained individuals.

\section{MRSA carriers}

Carriers of MRSA are asymptomatic (Figure 3). Clues to having a carrier would be repeated infections in your patients! The best way to find those carriers is to screen yourself and your staff (including those with minimal patient contact) via culture of nasal passage since the nose is the most commonly colonized mucous membrane area.

Factors that facilitate transmission between both asymptomatic carriers and infected patients include the following:

- $\quad$ Crowding or close proximity of individuals

- Antimicrobial use

- Contaminated surfaces and shared items

- Compromised skin

- $\quad$ Frequent contact

It goes without saying that most or all of these are factors in hair transplant surgery, and all of them can be controlled with proper vigilance.

\section{MRSA infection presentation and treatment}

MRSA will present in several different ways, including infections of the blood, lungs, joints, eyes, and urine. Admittedly, infections in these systems are less common and not as relevant to our field, but they bear mentioning for completeness sake. In hair surgery patient populations, infections usually present in otherwise healthy individuals as a skin infection (Figure 4). In hair surgery patients, look for the following:

- Clusters of sores (that resemble "spider bites")

- Impetigo (pus-filled blisters)

- Any incision that is erythematous, swollen, or has pus

- Erythematous painful boils or abscesses in donor or recipient areas $^{9}$

Infections that recur after one or more courses of antibiotics should immediately raise a red flag. Similarly, a cluster of patient infections without a clear source may indicate a staff source. The best course of action is to swab the infected area BEFORE BEGINNING TREATMENT WITH
FIGURE 4. Patient with suspected MRSA in donor area.

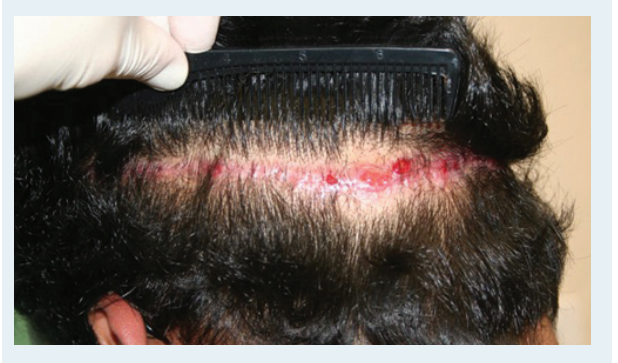


FIRST-LINE ANTIBIOTICS and send for culture and sensitivities.

This may be a change for practices that are used to starting empiric antibiotics, but it is important for two reasons. First, and most obvious, once the pathogen is known, antibiotic courses then can be tailored to the specific strain and avoid breeding further resistant bacteria. Second, in some countries, certain dangerous bacterial strains need to be reported to infection control authorities and knowing the pathogen involved allows infection control efforts to be more effective worldwide. We are all going to need to do our part if we are going to avoid the serious consequences of our current antibiotic prescribing habits.

One last point worth mentioning is that each practice should screen its patients for random antibiotic use, or incomplete antibiotic use. We are all human, and non-compliance with treatment is common (or even self-treatment with old, leftover, and inappropriate antibiotics). Simply ask the question as a matter of routine to all of your patients, particularly those presenting with any signs of infection.

\section{MRSA action steps}

It is easier to ignore these sobering facts and soldier on with our practices without changing any routine. Commonly, physicians consider their area "safe," and that MRSA is a problem in "other places." According to the World Health Organization (WHO), this belief is inaccurate. Their first global report on antibiotic resistance demonstrated soberingly high percentages of $\mathrm{S}$. aureus infections that were reported to be MRSA. That is why the first and best thing you can do to protect your practice and the future of our specialty is to educate yourself. ${ }^{10}$

$\begin{array}{ll}\text { WHO Region } & \% \text { Staph infections reported to be MRSA } \\ \text { African } & 80 \% \\ \text { Americas } & 90 \% \\ \text { Eastern Mediterranean } & >50 \% \\ \text { European } & 60 \% \\ \text { South-East Asia } & >25 \% \\ \text { Western Pacific } & 80 \%\end{array}$

(http://www.who.int/mediacentre/news/releases/2014/amr-report/en/)

Below are practical action steps you can take to protect your patients and staff from spreading or contracting MRSA:

1. Make a history of MRSA infection a part of your preoperative history. A surprising number of patients are now aware of their MRSA history and can alert you to their infectious potential if you ask.

2. Evaluate ALL members of your staff for MRSA-even those who might have minimal patient contact. This involves swabbing the noses of everyone including yourself, which may not sound enjoyable, but is preferable to dealing with an epidemic among your patients.

3. Avoid shaving the scalp. Various studies and meta-analyses paradoxically have shown that higher rates of infection occur with shaving than occur without. ${ }^{11}$

4. Discourage medical tourism. We all love it when our expertise is sought by patients from outside our usual catchment, and yet where possible, we should be en- couraging local treatment options. This reduces the risk or inadequate follow-up treatment for your patient and it also protects you and your staff from novel bacterial strains. Isolationism is economically unhealthy, and in the case of medical tourism, potentially the wiser course. ${ }^{7}$

5. Avoid unnecessary perioperative antibiotic use (and emphasize that your patients should take ALL of the antibiotics that you prescribe!). The guidelines for perioperative antibiotics are based on observed rates of infection and are different than we originally were taught in medical school. Not only is it interesting to read, it is our responsibility to keep up on this information, AND it will help you prep for your ABHRS Board Exam (or recertification!). Here are three references for directing your perioperative use of antibiotics:

A. Bratzler, D.W., et al. Clinical practice guidelines for antimicrobial prophylaxis in surgery. Am J Health-Syst Pharm. 2013; 70:195-283. (Also available at http://www.idsociety.org/Antimicrobial_Agents/).

This is a consensus document with over 1,000 references to back up its data, analyses, and suggestions.

B. http://ecdc.europa.eu/en/healthtopics/Healthcare-associated_infections/guidance-infection-prevention-control/Pages/ guidance-perioperative-antimicrobial-prophylaxis.aspx (European Centre for Disease Prevention and Control)

C. https://www.guideline.gov/summaries/summary/39533. This link is to the guidelines page from the National Guideline Clearinghouse. Another source with expert commentary, which is broken out into age group and surgical site for easy searchability.

The increasing frequency of bacterial infections with a MRSA strain mean that minor injuries could once again kill patients. Since we are in the business of creating minor injuries, it is our responsibility to know the facts and have a high level of suspicion for hair transplant surgery infections that could be MRSA.

\section{References}

1. For your reference, these are Clostridium Difficile, or CDIFF, Carbapenem-Resistant Enterobacteriaceae, or CRE, and Neisserie Gonorrhoeaea. (From the CDC's 2013 report-see reference \#2.)

2. https://www.cdc.gov/drugresistance/threat-report-2013/pdf/arthreats-2013-508.pdf \#page $=49$

3. http://www.handinscan.com/global-epidemiology-of-mrsa/

4. http://www.who.int/drugresistance/publications/infographic-antimicrobial-resistance-20140430.pdf?ua=1

5. See MRSA History Timeline: http://mrsa-research-center.bsd.uchicago.edu/timeline.html

6. http://www.cddep.org/tool/mrsa_infection_rates_country

7. Shah, S. Pandemic: Tracking Contagions from Cholera to Ebola and Beyond. Sarah Crichton Books, 2016.

8. https://www.cdc.gov/mrsa/community/index.html

9. http://www.health.state.mn.us/divs/idepc/diseases/mrsa/book.html (Minnesota Department of Health Website-a very good resource!)

10. http://www.who.int/mediacentre/news/releases/2014/amr-report/en/

11. Sebastian, S. Does preoperative scalp shaving result in fewer postoperative wound infections when compared with no scalp shaving? A systematic review. J Neurosci Nurs. 2012(Jun); 44(3):149-156. (Also available at https://www.ncbi.nlm.nih.gov/ pubmed/22555352.) 